[0212-7199 (2005) 22: 9; pp 419-423] ANALES DE MEDICINA INTERNA Copyright (C) 2005 ARAN EDICIONES, S.L.

An. Med InTERna (Madrid) Vol. 22, N. ${ }^{\circ}$ 9, pp. 419-423, 2005

\title{
Opinión sobre la libre elección de especialista por parte de pacientes diabéticos atendidos indistintamente por el equipo médico de Endocrinología de un hospital comarcal
}

\author{
C. MACIÁ BOBES, P. BOTAS CERVERO, A. RONZÓN FERNÁNDEZ1', A. ARANGO \\ FERNÁNDEZ
}

Sección de Endocrinología. Hospital San Agustín. Avilés. Asturias. ${ }^{1}$ Unidad de Medicina Familiar y Comunitaria. Hospital San Agustín. Avilés. Asturias

DIABETIC PATIENTS' CHOICE OF ENDOCRINOLOGIST IN A COUNTY HOSPITAL. OPINION OF PATIENTS WITHOUT POSSIBILITY OF FREE CHOICE

\section{RESUMEN}

Antecedentes: La libre elección de especialista en Endocrinología es un derecho de los usuarios de la sanidad pública española.

Objetivos: 1.- Averiguar si existe diferencia, en el contexto de un hospital comarcal español, en la opinión de los pacientes diabéticos vistos por primera vez en consulta y los que están en seguimiento prolongado respecto a la preferencia por la libre elección de especialista. 2.- Averiguar qué variables se asocian a esta preferencia entre los pacientes diabéticos en seguimiento.

Diseño: estudio transversal con entrevista directa.

Pacientes y métodos: 1.- Pacientes: Diabéticos tipo 1 y tipo 2, vistos en una primera consulta (grupo $\mathrm{A}, \mathrm{n}=60$ ), o con un seguimiento mínimo de 4 años (grupo $\mathrm{B}, \mathrm{n}=100$ ). Los pacientes fueron seleccionados de modo consecutivo en las consultas externas de Endocrinología. 2.- Métodos estadísticos: a) Descripción de las características de los pacientes y comparación entre grupos mediante las pruebas de Chi-cuadrado y U de Mann-Whitney. b) Objetivo 1: comparación mediante Chi-cuadrado. c) Objetivo 2: análisis mediante regresión logística multivariante.

Resultados: Objetivo 1: Existe una diferencia estadísticamente significativa entre los grupos A y B en la preferencia por la libre elección de especialista. Objetivo 2: Dentro del grupo B los pacientes con niveles más altos de hemoglobina glicosilada son los que prefieren elegir especialista en mayor medida.

Conclusiones: La libre elección de especialista es más apetecida por el grupo de pacientes diabéticos que opina "a priori" que por el grupo de pacientes que ha experimentado el sistema de atención en equipo. Dentro de este grupo sólo los niveles altos de hemoglobina glicosilada predicen la preferencia por la libre elección.

PALABRAS CLAVE: Libre elección de especialista. Pacientes diabéticos. Equipo médico de Endocrinología. Hospital comarcal.

\section{ABSTRACT}

Background: Free choice of specialized physicians is a patients'right in the Spanish Public Health Service.

Objectives: 1.- In diabetic outpatients visited in a county hospital to ascertain if there's any difference about endocrinologist free choice opinion between new and long-standing follow-up groups. 2.- In the second group of patients to study wich variables are associated with the favourable free choice opinion.

Design: Transversal, personal interview based.

Patients and methods: 1.- Type 1 and type 2 diabetic patients firstly (A group, $n=60$ ) or at least 4 years time of follow-up (B group, $n=100$ ) visited in an outpatient department of Endocrinology. 2.- Statistical methods: a) Description of groups and comparative analysis of their characteristics (Chi-squared and Mann-Whitney U tests). b) Objective 1: Chisquared test comparison. c) Objective 2: Multivariate logistic regression analysis.

Results: Objective 1: The possibility of free choice of endocrinologist is preferred by A-group patients in a statistically significant greater proportion than B-group patients. Objective 2: In the latest group subjects with higher levels of glycated haemoglobin are the more favourables to the free choice.

Conclusions: In a Spanish county hospital opinion about free choice of endocrinologist is better in an "a priori" judgement basis than after the personal experience of medical team attention. In long-standing follow-up patients only high levels of glycated haemoglobin predict the preference for the free choice.

KEY WORDS: Physician's patient free choice. Diabetic patients. Endocrinological medical team. County hospital.

Maciá Bobes C, Botas Cervero P, Ronzón Fernández, A, Arango Fernández. A. Opinión sobre la libre elección de especialista por parte de pacientes diabéticos atendidos indistintamente por el equipo médico de Endocrinología de un hospital comarcal. An Med Interna (Madrid) 2005; 22: 419-423.

\section{INTRODUCCIÓN}

Dentro de la red sanitaria pública española, la libre elección de endocrinólogo en consultas externas es un derecho de los pacientes sustanciado en el Real Decreto 8/1996, de 15 de enero (1), y aplicable en el territorio del antiguo Instituto Nacional de la Salud. Sin embargo, en algunos modelos de citación puede resultar difícil garantizar este derecho, especialmente en hospitales comarcales con plantillas reducidas y en los que el tiempo dedicado a la consulta ambulatoria tiende

Trabajo aceptado: 27 de mayo de 2005 
a ser el máximo posible (lo cual resulta poco compatible con la asignación de días concretos de consulta para cada médico).

En el ámbito de la atención primaria existen múltiples estudios, tanto españoles (2-5) como de otros países (6-11), sobre la asociación positiva entre la posibilidad de escoger médico y la satisfacción de los usuarios con la asistencia recibida. Por supuesto esta libre elección no es el único factor determinante de la satisfacción $(2,3,5,6)$. Sin embargo los estudios equivalentes en atención especializada son pocos y no proceden de grupos españoles (12-16), con la consiguiente dificultad para extrapolar resultados obtenidos en países (básicamente EE.UU.) con sistemas sanitarios muy diferentes al nuestro.

En el hospital San Agustín (Avilés, Asturias), que atiende un área sanitaria de 160.000 personas, la Sección de Endocrinología está constituida por tres facultativos de plantilla, además del personal de enfermería. No existe una personalización de las agendas de consultas, de tal manera que cualquier paciente, tanto en la primera cita como en las sucesivas, puede ser asignado a cualquier especialista. La razón es que cada día funcionan $(\mathrm{x}-0,5)$ consultas, donde $\mathrm{x}=$ número de médicos en activo y donde 1 consulta equivale a 7 horas. Resulta inviable en la práctica determinar con meses de antelación qué facultativo/s se hará/n cargo de la/s consulta/s un día concreto. El resultado es que la mayoría de los pacientes diabéticos (y no diabéticos) con un seguimiento medianamente prolongado ha sido visto por los tres médicos. Esta circunstancia no ha generado conflictos (quejas verbales en la consulta o quejas formales en el servicio de Atención al Paciente) salvo de modo completamente excepcional.

Los objetivos de este estudio son dos:

1. Averiguar si existe diferencia en la opinión (positiva o no-positiva) de los pacientes diabéticos (crónicos por excelencia) vistos en una primera consulta y los que están en seguimiento prolongado, sobre la posibilidad de una libre elección de especialista, definida como la garantía de acceso al mismo o a diferente médico, en función de los deseos del paciente.

2. Averiguar qué variables se asocian a la preferencia por la libre elección (opinión positiva) entre los pacientes diabéticos en seguimiento.

\section{PACIENTES Y MÉTODOS}

\section{PACIENTES}

Fueron seleccionados de modo consecutivo entre los que acudieron a la consulta externa de Endocrinología del hospital San Agustín (Avilés, Asturias) en un período de tiempo de 3 meses. No hubo criterios de exclusión. En el grupo A se incluyeron 60 pacientes diabéticos, tipo 1 y tipo 2 (predominantemente tipo 2 dada la prevalencia de ambas formas), vistos en primera consulta. De las 100-120 primeras consultas mensuales realizadas aproximadamente un $20-30 \%$ corresponden a diabetes (DM). En el grupo B se incluyeron 100 pacientes diabéticos, tipo 1 y tipo 2 (en proporción similar de acuerdo con el perfil de "retención" para seguimiento), vistos al menos 2 veces por cada uno de los 3 endocrinólogos de plantilla en el curso de los 4 últimos años. De modo habitual las revisiones médicas diabéticas se programan con frecuencia semestral; se calculó que un 30-40\% de los 100-120 pacientes diabéticos que se siguen mensualmente en la consulta cumplirían el cri- terio de inclusión. La cifra de 100 se obtuvo de la fórmula 10 $\mathrm{x}(\mathrm{k}+1)$, donde $\mathrm{k}=9$ fue el número de variables independientes pre-seleccionadas para la regresión logística.

En la consulta médica, de modo directo, se formuló a los pacientes la pregunta: "Si pudiera elegir ¿preferiría ser visto siempre por el mismo endocrinólogo?", con la explicación de que inicialmente se les asignaría el que les tocase al azar en la primera consulta (antes de la cual la elección constituiría un pre-juicio), pero con posibilidad de cambio si la relación no era óptima (extremo bajo decisión del paciente). En realidad se trataría de garantizar la "continuidad" del facultativo a cargo, y también de salvaguardar la posibilidad de elegir. Ningún paciente se negó a contestar. La respuesta a la pregunta (variable dependiente) se tabuló como 0: Respuesta negativa (no preferencia) y 1: Respuesta positiva (preferencia).

\section{MÉTODOS ESTADÍSTICOS}

La descripción de las características de los pacientes, tomadas de la historia clínica, se efectuó en forma de porcentajes para las variables categóricas y en forma de media, mediana e intervalo de confianza para la media (con seguridad del 95\%) para las variables continuas. No se asumió una distribución normal de estas variables, aspecto que se comprobó mediante el test de Kolmogorov-Smirnov. La comparación entre grupos, para las características que podían ser sistemáticamente recogidas en la primera consulta, se efectuó mediante la prueba de Chi-cuadrado (variables categóricas) o la U de Mann-Whitney (variables continuas).

Para responder al objetivo número 1 del trabajo se procedió a una comparación de proporciones de la variable dependiente, mediante Chi-cuadrado, entre los grupos A y B.

Para responder al objetivo número 2 se realizó en primera instancia una categorización de las variables independientes pre-seleccionadas $(\mathrm{k}=9)$ : sexo, edad, tipo de diabetes, tiempo de evolución de la diabetes, tiempo de seguimiento en la consulta, nivel de estudios, hábitat, control glucémico (hemoglobina glicosilada -HbA1c- media del último año) y presencia de microangiopatía diabética (retinopatía y/o nefropatía diabéticas).

A continuación se efectuó un estudio de regresión logística multivariante $(17,18)$ en el que se partió de un modelo máximo, con inclusión de todas las variables, y se procedió luego a una eliminación hacia atrás paso a paso. En el modelo final se conservaron aquéllas cuya significación en el contraste de la razón de verosimilitud fue inferior a 0,15. La bondad de ajuste de este modelo se calculó mediante la prueba de Chicuadrado.

En todos los casos la significación estadística se fijó para una $\mathrm{p}<0,05$ en el contraste bilateral. Para el cálculo de los estadísticos se utilizó el programa SPSS, versión 11.0 para Windows.

\section{RESULTADOS}

En la tabla I se describen las características de los pacientes de los grupos A y B. Puesto que la distribución de las variables continuas no fue normal se proporciona la mediana, además de la media y el intervalo de confianza al 95\% para la media. El tiempo de seguimiento en consulta no era tributario 
TABLA I

CARACTERÍSTICAS DE LOS PACIENTES

\begin{tabular}{lcc}
\hline & $\begin{array}{c}\text { Grupo } A \\
(N=60)\end{array}$ & $\begin{array}{c}\text { Grupo } B \\
(N=100)\end{array}$ \\
\hline ISexo (M/F): $n / n$ (\%) & $25 / 35(41,7 / 58,3)$ & $50 / 50(50,0 / 50,0)$ \\
Edad (años): media/mediana (IC 95) & $60,1 / 61,0(56,5-63,6) \&$ & $52,1 / 55,0(48,9-55,3)$ \\
Hábitat (U/R): $n / n(\%)$ & $47 / 13(78,3 / 21,7)$ & $68 / 32(68,0 / 32,0)$ \\
Estudios (P/S-U): $n / n(\%)$ & $47 / 13(78,3 / 21,7) \& \&$ & $62 / 38(62,0 / 38,0)$ \\
Tipo de diabetes (1/2): $n / n$ (\%) & $5 / 55(8,3 / 91,7) \& \&$ & $45 / 55(45,0 / 55,0)$ \\
Tiempo de evolución de la diabetes (años): media/mediana (IC 95) & $10,3 / 7,0(7,7-12,8) \&$ & $16,5 / 15,0(14,5-18,5)$ \\
Tiempo de seguimiento en consulta: media/mediana (IC 95) & & $8,4 / 8,0(7,7-9,1)$ \\
HbA1c media del último año (\%): media/mediana (IC 95) & & $7,6 / 7,4(7,4-7,8)$ \\
Presencia de microangiopatía diabética (No/Sí): $n / n$ (\%) & & $54 / 46(54,0 / 46,0)$ \\
\hline
\end{tabular}

Grupo A: Pacientes diabéticos vistos en consulta por primera vez; Grupo B: Pacientes diabéticos en seguimiento (mínimo de 4 años); IC 95: Intervalo de confianza para la media al 95\%; M/F: Masculino/Femenino; U/R: Urbano/Rural; P/S-U: Primarios o menos/Secundarios o universitarios; HbA1c: Hemoglobina glicosilada; Microangiopatía diabética: retinopatía y/o nefropatía diabéticas; $\&=p<0,05$ (U de Mann-Whitney) (grupo A vs. grupo B); $\& \&=p<0,05$ (Chi-cuadrado) (grupo A vs. grupo B).

de contraste entre grupos, y ni la HbA1c previa ni la presencia de microangiopatía diabéticas eran datos sistemáticamente disponibles en los pacientes del grupo A. El resto de los parámetros se comparó entre grupos mediante pruebas no paramétricas. Existió una diferencia significativa en cuanto a la edad, el nivel de estudios, el tipo de DM, y el tiempo de evolución de la DM.

En la tabla II se efectúa la comparación entre grupos respecto a la preferencia por la libre elección, que resulta significativamente menor (para un menor número de pacientes es importante poder decidir) en el grupo B que en el grupo A. La significación estadística se consigue a pesar de la diferencia numérica entre los grupos (100 vs. 60).

En la tabla III se describe la categorización de las 9 variables independientes consideradas con vistas a la entrada en el análisis de regresión logística.

En la tabla IV se resume la estimación de los parámetros más relevantes del análisis de regresión logística. Se proporcionan datos de las tres variables retenidas. La única predictora de preferencia por la libre elección que se mantuvo en el modelo final fue el nivel de HbA1c medio del último año: los pacientes con peor $\mathrm{HbA} 1 \mathrm{c}$ desearon ser vistos siempre por el mismo especialista en proporción significativamente mayor.

\section{TABLA II}

COMPARACIÓN ENTRE GRUPOS (A Y B) RESPECTO A LA LIBRE ELECCIÓN DE ESPECIALISTA

$\begin{array}{cc}\text { Grupo } A & \text { Grupo } B \\ (N=60) & (N=100)\end{array}$

Preferencia por la libre elección

$\begin{array}{lcc}\text { NO: } n(\%) & 19(31,7) & 61(61,0) \\ \text { SI: } n(\%) & 41(68,3) & 39(39,0) \\ \text { Significación estadística } & p<0,001 \text { (Chi-cuadrado) }\end{array}$

Grupo A: Pacientes diabéticos vistos en consulta por primera vez; Grupo B: Pacientes diabéticos en seguimiento (mínimo de 4 años).

\section{DISCUSIÓN}

Los pacientes de los grupos A y B que se comparan para dar respuesta al primero de los objetivos de este estudio no son equiparables en varios aspectos clínicos: los del grupo A (reclutados en una primera visita) son de mayor edad (y probablemente por esta razón su nivel de estudios es menor), han sido diagnosticados de DM hace menos tiempo y son prácticamente todos tipo 2. Estas diferencias, que apuntan a una menor dificultad en el manejo de la DM, son esperables si se considera que el perfil del paciente diabético "retenido" en la consulta especializada debe de ser más complejo que el devuelto a atención primaria. El estudio no está diseñado para que el grupo A sea representativo de los pacientes con DM seguidos en primaria y en algún momento derivados a atención especializada, ni para que el grupo B sea representativo de los diabéticos en seguimiento por la consulta externa de Endocrinología. Por otra parte ninguna de las variables cuya diferencia entre grupos fue estadísticamente significativa resultó luego predictora de la opinión sobre la libre elección.

El número de pacientes que integran el grupo A (60) fue el reclutado en el mismo período (3 meses) que se necesitó para reclutar los 100 del grupo B, cifra pre-establecida en función del número de variables independientes inicialmente consideradas. Si el contraste entre grupos no hubiera resultado estadísticamente significativo, lo cual constituía la hipótesis de trabajo, se hubiera aumentado el número del grupo A para dar más potencia a la prueba estadística.

En números redondos, para un $32 \%$ de pacientes "nuevos" y un $61 \%$ de pacientes en seguimiento la posibilidad de la libre elección, según la hemos definido, no es importante, hasta el punto de no preferirla en respuesta a una pregunta neutra. Las dos cifras, pero particularmente la segunda, hacen pertinente la duda de si la libre elección de especialista, consagrada legalmente "dentro del proceso de mejora de la organización y de la calidad de los servicios sanitarios" (...), según se afirma textualmente en el Real Decreto (1), pero mucho más probablemente para satisfacer el deseo de los pacientes, representará una mejora subjetivamente percibida como tal por los 
TABLA III

CATEGORIZACIÓN DE VARIABLES PARA LA REGRESIÓN LOGÍSTICA (PREDICCIÓN DE LA PREFERENCIA POR LA LIBRE ELECCIÓN DE ESPECIALISTA EN EL GRUPO B)

Variable dependiente: Opinión positiva o negativa a la pregunta: "¿Preferiría ser visto siempre por el mismo endocrinólogo?"

0 : Respuesta negativa

1: Respuesta positiva

Variables independientes $(k=9)$

\begin{tabular}{lcc}
\hline & Masculino & Categorización \\
*Sexo & Femenino & 1 \\
& $<40$ & 2 \\
*Edad (años) & $40-60$ & 1 \\
& $>60$ & 2 \\
*Tipo de diabetes & 1 & 3 \\
& 2 & 1 \\
*Tiempo de evolución & $<10$ & 2 \\
de la diabetes (años) & $10-20$ & 1 \\
& $>20$ & 2 \\
*Seguimiento (años) & $<5$ & 3 \\
& $5-10$ & 1 \\
& $>10$ & 2 \\
*Nivel de estudios & Primarios o menos & 3 \\
& Secundarios o universitarios & 2 \\
*Hábitat & Urbano & 1 \\
& Rural & 2 \\
*Control (HbA1c media & $\leq 7,5 \%$ & 1 \\
del último año) (\%) & $>7,5$ & 2 \\
*Presencia de & No & 0 \\
microangiopatía diabética & Sí & 1
\end{tabular}

Grupo B: Pacientes diabéticos en seguimiento (mínimo de 4 años); HbA1c: Hemoglobina glicosilada; Microangiopatía diabética: retinopatía y/o nefropatía diabéticas.

usuarios de las consultas externas de los hospitales. No se concibe en qué pueda mejorar la organización (de los servi- cios sanitarios) la libre elección, y la mejora de la calidad sólo puede interpretarse como la percibida por el usuario.

Por supuesto, las respuestas obtenidas en este trabajo no son extrapolables a otros pacientes, otros Servicios u otros hospitales (12). Cabe imaginar cómo serían las recogidas en Servicios cuyos miembros mostrasen una notoria dispersión en sus actitudes diagnósticas o terapéuticas, o en su grado de amabilidad o empatía con los pacientes. Sin embargo, tanto los postulados de la Medicina Basada en la Evidencia (o en las pruebas), como el buen criterio común, o la defensa eficaz contra las posibles reclamaciones de los pacientes, abogan por minimizar en lo posible la variabilidad de la práctica clínica, con la implantación de protocolos y guías de práctica clínica, de vías clínicas, y de actuaciones basadas en las mejores pruebas disponibles $(19,20)$.

La buena (u óptima incluso) relación con los pacientes es menos sistematizable (4) y su variabilidad justificaría el remanente de usuarios que prefiere encontrarse siempre en la consulta ante el mismo especialista. Pero se trata, al menos en nuestra experiencia, de un "desideratum" no irrenunciable por cuanto la vía para defenderlo (el Servicio de Atención al Paciente) se utiliza de modo anecdótico. En nuestro caso, un sistema de citación con agendas personalizadas para cada médico, ocasionaría una necesidad de bloqueos muy superior a la originada por el sistema actual (citación de 2,5 y 1,5 consultas diarias, de modo alterno), y disfunciones por aperturas tardías que se traducirían en un incremento de las listas de espera. En nuestra opinión, en Secciones con alta saturación asistencial en consultas externas una implantación rigurosa del programa de libre elección de especialista podría ocasionar más perjuicio que beneficio.

La segunda parte del trabajo está dirigida a identificar los factores que pueden predecir la preferencia por la libre elección en el grupo de pacientes en seguimiento, del que se dispone de datos sobre variables presuntamente relacionadas. La pre-selección de estas variables se efectuó según opinión discrecional del equipo de Endocrinología. La única predictora fue el grado de control glucémico, expresado como la media de HbA1c del año anterior. Los pacientes con peor control eran, como grupo, los más partidarios de la elección.

Una lectura posible, no única, es la siguiente. El control de la DM depende de varias "familias" de factores $(21,22)$ : una se refiere a las características de la propia DM (tipo, años de

TABLA IV

MODELO FINAL DE REGRESIÓN LOGÍSTICA DE VARIABLES PARA LA PREDICCIÓN DE LA PREFERENCIA POR LA LIBRE ELECCIÓN DE ESPECIALISTA EN EL GRUPO B

Estimaciones de los parámetros

\begin{tabular}{|c|c|c|c|c|c|c|c|c|}
\hline \multirow[t]{2}{*}{ Preferencia por la libre elección } & & \multirow[t]{2}{*}{$B$} & \multirow[t]{2}{*}{$S E$} & \multirow[t]{2}{*}{ Wald } & \multirow[t]{2}{*}{ Sig } & \multirow[t]{2}{*}{$\operatorname{Exp}(B)$} & \multicolumn{2}{|c|}{ IC 95\% $\operatorname{Exp}(B)$} \\
\hline & & & & & & & $\begin{array}{l}\text { Limite } \\
\text { inferior }\end{array}$ & $\begin{array}{l}\text { Límite } \\
\text { superior }\end{array}$ \\
\hline & Sexo &,- 455 &, 458 & ,988 &, 320 &, 635 &, 259 & 1,556 \\
\hline & Hábitat &,- 925 &, 505 & 3,358 &, 067 & ,397 &, 147 & 1,066 \\
\hline & $\mathrm{HbA} 1 \mathrm{c}$ & 1,472 &, 454 & 10,535 &, 001 & 4,359 & 1,792 & 10,603 \\
\hline
\end{tabular}

Grupo B: Pacientes diabéticos en seguimiento (mínimo de 4 años); HbA1c: hemoglobina glicosilada; B: coeficiente de regresión; SE: Error estándar de B; Sig: Significación de B a través del estadístico de Wald; Exp (B): Exponenciales de B (odds ratio de cada variable independiente); IC $95 \%$ : Intervalos de confianza de Exp (B) al $95 \%$; En subrayado significación con $p<0,05$; Ajuste del modelo: Chi-cuadrado: 14,031, p=0,003 (3 grados de libertad). 
evolución, labilidad intrínseca, etc.), otra se refiere al contexto social y a la coexistencia de otras enfermedades y tratamientos, una tercera a la implicación del paciente en el objetivo de conseguir un buen control y al menos queda por mencionar la atención sanitaria recibida. Neutralizada la última, sólo la anterior resulta modificable; la consecuencia es que en nuestra Unidad el grado de presión ejercido sobre los pacientes (en forma de recomendaciones y advertencias), se relaciona de modo directo con la valoración médica de sus posibilidades individuales de mejora. Los pacientes peor controlados (en principio por cualquiera de las tres primeras causas) son, en nuestro medio, los que prefieren enfrentarse siempre al mismo facultativo, quizá porque una parte de ellos confía en que la intensidad de la presión sea así menor o como mínimo menos persistente. Se trataría en suma de una cuestión de comodidad y de evitación de entrevistas subjetivamente percibidas como estresantes por parte del grupo de pacientes tributarios de presión.

Como conclusión, en este estudio realizado por la Sección de Endocrinología del hospital San Agustín (Avilés, Asturias), la libre elección de especialista, definida como continuidad de la asistencia a cargo del médico escogido, es más apetecida por el grupo de pacientes diabéticos que opina "a priori" que por el grupo de pacientes que ha experimentado el sistema de atención en equipo. Dentro de este grupo sólo los niveles altos de hemoglobina glicosilada predicen la preferencia por la libre elección.

\section{Bibliografía}

1. Real Decreto 8/1996, de 15 enero, Ministerio de Sanidad y Consumo. BOE 31 enero 1996, núm. 27, pág. 2903 y siguientes.

2. Gómez Castro MJ, Bernal Blasco I, Guerra Cabrera F, Mirabal Sánchez V, Lorenzo Riera A. Satisfacción de los usuarios de una zona de salud. Diferencias según modelo asistencial. Aten Primaria 1997; 20: 90-3.

3. Bolíbar I y Grupo de Estudio de la Utilización de Servicios Sanitarios de Mataró. Determinantes poblacionales de la satisfacción con el médico de atención primaria. Gaceta Sanitaria 1999; 13: 371-83.

4. Torío Durántez J, García Tirado MC. Relación médico-paciente y entrevista clínica (I): opinión y preferencias de los usuarios. Aten Primaria 1997; 19: 18-26.

5. Delgado A, Martínez-Cañavate T, García V, Frías J, Rueda T, Morata J. Preferencias y estereotipos de los pacientes sobre el género del médico de familia. Aten Primaria 1999; 23: 268-74.

6. Gross R, Tabenkin H, Porath A, Heymann A, Greentein M, Porter B et al. The relationship between primary care physicians' adherence to guidelines for treatment of diabetes and patient satisfaction: findings from a pilot study. Fam Pract 2003; 20: 563-9.

7. Krupat E, Stein T, Selby JV, Yeager CM, Schmittdiel J. Choice of a primary care physician and its relationship to adherente among patients with diabetes. Am J Manag Care 2002; 8: 777-84.

8. Kersnik J. Determinants of customer satisfaction with the health care system, with the possibility to choose a personal physician and with a family doctor in a transition country. Health Policy 2001; 57: 155-64.

9. Schmittdiel J, Selby JV, Grumbach K, Quesenberry CPJr. Choice of a personal physician and patient satisfaction in a health maintenance organization. JAMA 1997; 278: 1596-9.

10. Kalda R, Polluste K, Lember M. Patient satisfaction with care is associated with personal choice of physician. Health Policy 2003; 64: 5562 .
11. Hsu J, Schmittdiel J, Krupat E, Stein T, Thom D, Fireman B, Selby J. Patient choice. A randomized controlled trial of provider selection. $\mathrm{J}$ Gen Intern Med 2003; 18: 319-25.

12. Hill CJ, Garner SJ. Factors influencing physician choice. Hosp Health Serv Adm 1991; 36: 491-503.

13. Hanna N, Schoenbachler DD, Gordon GL. Physician choice criteria: factors influencing patient selection of generalists versus specialists. Healtlh Mark Q 1994; 12: 29-42.

14. Kao AC, Green DC, Davis NA, Koplan JP, Cleary PD. Patients' trust in their physicians: effects of choice, continuity, and payment method. J Gen Intern Med 1998; 13: 681-6.

15. Harris, KM. How do patients choose physicians? Evidence from a national survey of enrollees in employment-related health plans. Health Serv Res 2003; 38: 711-32.

16. Cheng SH, Song HY. Physician performance information and consumer choice: a survey of subjects with the freedom to choose between doctors. Qual Saf Health Care 2004; 13: 98-101.

17. Silva LC. Excursión a la regresión logística en ciencias de la salud. Madrid; Díaz de Santos, 1994: 3-11.

18. Domínguez Alonso A, Aldana Padilla D. Regresión logística. Un ejemplo de su uso en Endocrinología. Rev Cubana Endocrinol 2001; 12: 58-64.

19. Evidence-based Medicine Working Group. Evidence-based medicine: a new approach to teaching the practice of medicine. JAMA 1992; 268 : 2493-8.

20. Concato J, Shah N, Horwitz RI. Randomized, controlled trials, observational studies, and the hierarchy of research designs. N Engl J Med 2000; 342: 1887-92

21. Peralta G, Figuerola D. Aspectos psicosociales de la diabetes. Endocrinol Nutr 2003; 50: 280-5.

22. Vázquez Martínez C, Canovas B, Jaunsolo MA. Educación diabética y automonitorización. Guías de actuación clínica de la diabetes mellitus. Endocrinol Nutr 2003; 50 (Supl. 1): 61-4. 